\title{
Expectation and temperament moderate amygdala and dorsal anterior cingulate cortex responses to fear faces
}

\author{
Jacqueline A. Clauss $\cdot$ Ronald L. Cowan • \\ Jennifer Urbano Blackford
}

Published online: 16 November 2010

(C) The Psychonomic Society, Inc. 2010

\begin{abstract}
A chronic tendency to avoid novelty is often the result of a temperamental bias called inhibited temperament, and is associated with increased risk for anxiety disorders. Neuroimaging studies have demonstrated that an inhibited temperament is associated with increased amygdalar blood-oxygenation-level-dependent (BOLD) response to unfamiliar faces that were not expected; however, the effects of variations in expectancy remain unknown. Using functional magnetic resonance imaging (fMRI), we studied BOLD response to infrequently encountered fear faces that were either expected or not expected in 42 adults with an inhibited or an uninhibited temperament. Individuals with an inhibited temperament had greater amygdala, but less dorsal anterior cingulate cortex (dACC), BOLD response when the stimuli were expected. In contrast, those with an uninhibited temperament had a smaller amygdala but larger dorsal anterior cingulate cortex BOLD response when expecting to see fear faces. These findings demonstrate temperament differences in
\end{abstract}

Electronic supplementary material The online version of this article (doi:10.3758/s13415-010-0007-9) contains supplementary material, which is available to authorized users.

\footnotetext{
J. A. Clauss $\cdot$ R. L. Cowan $\cdot$ J. U. Blackford $(\bowtie)$

Vanderbilt Medical School Department of Psychiatry,

1601 23rd Avenue South, Suite 3057J,

Nashville, TN 37212, USA

e-mail: Jennifer.Blackford@,Vanderbilt.edu

URL: www.blackfordlab.com

J. A. Clauss

e-mail: Jacqueline.A.Clauss@Vanderbilt.edu

R. L. Cowan · J. U. Blackford

Vanderbilt Department of Psychology,

Nashville, TN, USA

R. L. Cowan

Vanderbilt Department of Radiological Sciences,

Nashville, TN, USA
}

expectancy effects and provide preliminary evidence for the $\mathrm{dACC}$ as a neural substrate mediating differences in inhibited temperament. Enhanced amygdala sensitivity coupled with weak inhibitory control from the AACC may form a neural circuit mediating behaviors characteristic of inhibited temperament and risk for anxiety disorders.

Keywords Temperament · fMRI - Expectancy · Amygdala · Dorsal anterior cingulate cortex

\section{Introduction}

The brain rapidly distinguishes between new and old stimuli, which is adaptive when animals must process large amounts of sensory information (Ranganath \& Rainer, 2003). Although novelty detection is universal, novelty evokes widely different behaviors across individuals. The chronic tendency to avoid novel events and situations characterizes those with behavioral inhibition or an "inhibited temperament" (Kagan, Reznick, Snidman, Gibbons, \& Johnson, 1988b). Inhibited temperament is defined as the biologically-based predisposition to respond to novel object, places, and objects with wariness or avoidance behaviors. Individuals with an inhibited temperament are shy, cautious, and restrained, whereas those who are uninhibited are more often outgoing, adventurous, and spontaneous. Approximately $15 \%$ of infants are born with an inhibited temperament (Kagan, Reznick, \& Snidman, 1988a), and the trait is relatively stable across development (Kagan, Reznick, \& Snidman, 1987; Goodwin, Fergusson, \& Horwood, 2004; Windle \& Windle, 2006; Kagan et al., 1987). Inhibited temperament has robust physiological correlates including a high heart rate with low variability (Kagan et al., 1988a) and greater right frontal EEG asymmetry (Calkins, Fox, \& 
Marshall, 1996; Fox, Henderson, Rubin, Calkins, \& Schmidt, 2001). Children with an inhibited temperament are at increased risk for anxiety disorders (Kagan, Snidman, Zentner, \& Peterson, 1999; Schwartz, Snidman, \& Kagan, 1999), with the risk highest for those children that continue to be inhibited throughout adolescence (Chronis-Tuscano et al., 2009; Essex, Klein, Slattery, Goldsmith, \& Kalin, 2010; Hirshfeld et al., 1992). Identifying the functional brain differences underlying an inhibited temperament will enhance our understanding of these behavioral biases and how they modulate the risk for anxiety disorders.

The amygdala has been regarded as an important structure in the development of an inhibited temperament (Kagan et al., 1988b). The amygdala reliably responds to all novelty, including novel faces (Schwartz et al., 2003b; Wright et al. 2003; Zald, 2003) and scenes (Blackford, Buckholtz, Avery, $\&$ Zald, 2010). Both timing and magnitude of amygdalar blood-oxygenation-level-dependent (BOLD) responses differ by temperament, with those possessing an inhibited temperament producing both faster (Blackford, Avery, Shelton, \& Zald, 2009) and larger (Schwartz, Wright, Shin, Kagan, \& Rauch, 2003a) responses to novel faces. In inhibited individuals, amygdala BOLD response is sustained over time when viewing newly familiarized faces; that is the amygdala fails to show the typical pattern of habituation seen in individuals with an uninhibited temperament (Blackford, Avery, Cowan, Shelton, \& Zald, in press). While these studies provide initial support for the amygdala as a neural substrate of inhibited temperament, previous studies focused on only one brain region, and one origin of novelty. But, the term novel can refer to an event that is unfamiliar, or one that is unexpected.

Expectation plays a critical role in information processing, with past and current information constantly used to predict future events (Bubic, von Cramon, \& Schubotz, 2010). Differences in expectancies engage the amygdala (Belova, Paton, Morrison, \& Salzman, 2007; Bermpohl, Pascual-Leone, Amedi et al., 2006; Johansen, Tarpley, LeDoux, \& Blair, 2010; Onoda et al., 2008). Given the key role of the response to novelty in inhibited temperament, one would predict that these individuals would also be affected by variation in expectancy. However, differences in expectancy could produce either increased amygdala BOLD response to the unexpected events or increased amygdala BOLD response to the expected events in those with an inhibited temperament. Based on the definition of inhibited temperament and the previous findings of increased amygdala BOLD response to novel or newly familiar faces (Blackford et al., 2009; Blackford et al., in press; Schwartz et al., 2003a), one might predict increased amygdala BOLD response to the unexpected, or novel, events. This finding would provide converging evidence for the theory that a hyper-excitable amygdala underlies an inhibited temperament. However, another prediction is equally plausible. Prior knowledge of an upcoming negative event may increase vigilance for that negative event. Given the conceptualization of inhibited temperament, we would predict that these individuals to demonstrate enhanced vigilance relative to those with an uninhibited temperament. A biological basis of increased vigilance may be an increased signalto-noise ratio in neural structures specialized for the processing of threatening stimuli (Foote, Bloom, \& Astonjones, 1983). Specifically, under conditions of vigilance, neural responses may be potentiated (Whalen, 1998), resulting in a larger amygdala BOLD response to the expected negative event. Our goals for this study were to examine temperament differences in expectancy effects and to search for additional neural correlates of inhibited temperament.

\section{Methods}

Subjects

Forty-two adults, with either an inhibited $(n=20 ; 12$ females, eight males) or an uninhibited ( $n=22 ; 12$ females, ten males) temperament, participated in the study. Subjects had an average age of 21 years ( $\mathrm{SD}=0.9)$, were predominantly right-handed $(93 \%)$, and represented a variety of ethnic groups (64\% Caucasian, non-Hispanic, 12\% Caucasian, Hispanic, 14\% African-American, 5\% Asian-American, 2\% other). Inhibited and uninhibited subjects did not differ on age, gender, handedness, or ethnicity (see Table 1). Five subjects were removed from the analysis due to image quality (see Methods), resulting in a final analytic sample size of 37 subjects (18 inhibited, 19 uninhibited). The Vanderbilt University Institutional Review Board approved the study and we obtained written informed consent after providing subjects with a complete description of the study.

Subject selection Subjects were recruited for a larger study of temperament by placing advertisements seeking persons who were "especially shy or outgoing as a child". Interested individuals completed two screening questionnaires measuring childhood temperament and adult temperament. Childhood temperament was assessed using the Retrospective Self-Report of Inhibition (RSRI), a 30-item measure that asks about childhood behaviors on a 1-5 Likert scale $(1=$ uninhibited, $5=$ inhibited $)$. The companion measure, the Current Self-Report of Inhibition (CSRI), was used to assess adult temperament (31 items; $1-5$ Likert scale). The RSRI and CSRI have good reliability and construct validity in unselected samples (Cronbach's alpha RSRI $=.79$, ASRI $=.77$; Reznick, Hegeman, Kaufman, Woods, \& 
Table 1 Subject characteristics

\begin{tabular}{|c|c|c|c|c|c|}
\hline \multirow[b]{2}{*}{ Temperament } & \multicolumn{2}{|c|}{ Inhibited Temperament } & \multicolumn{3}{|c|}{ Uninhibited Temperament } \\
\hline & Mean & SD & Mean & SD & $p$ value \\
\hline Retrospective & 3.06 & 0.11 & 1.48 & 0.07 & $<.001$ \\
\hline Current & 3.02 & 0.07 & 1.59 & 0.06 & $<.001$ \\
\hline \multicolumn{6}{|l|}{ Demographics } \\
\hline \multirow[t]{2}{*}{ Age (Years) } & 24.7 & 1.1 & 22.2 & 0.6 & $\mathrm{~ns}$ \\
\hline & Percent & $\mathbf{N}$ & Percent & $\mathbf{N}$ & $p$ value \\
\hline Gender (\% Female) & $64 \%$ & 12 & $55 \%$ & 12 & ns \\
\hline Handedness (\% Right) & $75 \%$ & 15 & $64 \%$ & 14 & ns \\
\hline \multicolumn{6}{|l|}{ Ethnicity } \\
\hline$\%$ Caucasian & $80 \%$ & 16 & $73 \%$ & 16 & ns \\
\hline$\%$ African American & $15 \%$ & 3 & $18 \%$ & 4 & ns \\
\hline$\%$ Asian & $5 \%$ & 1 & $5 \%$ & 1 & ns \\
\hline$\%$ Other & $0 \%$ & 0 & $5 \%$ & 1 & \\
\hline
\end{tabular}

$\mathrm{ns}=$ not significant

Jacobs, 1992) and excellent reliability in this sample (Cronbach's alpha RSRI $=.97$, ASRI $=.97$ ). We used normative guidelines (Reznick et al., 1992) to define cutoffs for extreme (top and bottom 15\%) temperament scores on both of the temperament questionnaires. The use of two extreme groups is based on the original description of inhibited temperament and is commonly used in studies of this temperament trait (Blackford et al., 2009; Guyer et al., 2006; Perez-Edgar et al., 2007; Schwartz et al., 2003a; BarHaim et al., 2009; Beaton et al., 2008). In addition, the extreme groups approach (Nebert, 2000) provides two homogenous and distinguishable behavioral profiles, which maximizes the likelihood of discovering differences in underlying brain function. Furthermore, we specifically recruit individuals with a stable pattern of inhibition (or lack thereof) because individuals who were inhibited as both children and still as adults are a more homogenous group than those that were only inhibited as children; and individuals who are still inhibited as adults are at greater risk for anxiety disorders compared to those who are no longer inhibited (Chronis-Tuscano et al., 2009; Essex et al., 2010; Hirshfeld et al., 1992).

For this study, we enrolled subjects who: were between 18 and 30 years of age; had extreme retrospective and current temperament scores; passed an MRI safety screen; and were free of psychoactive medications, substance abuse during the past six months, major medical illness, and history of brain trauma. Subjects were not excluded for psychiatric illness because inhibited temperament is associated with increased rates of anxiety. Psychiatric status was assessed by a trained clinical interviewer using the Structured Clinical Interview for DSM-IV (Spitzer, Williams, Gibbon, \& First, 1992). Consistent with other studies (Blackford et al., 2009; Schwartz et al., 1999), inhibited subjects had higher rates of current internalizing disorders compared to the uninhibited group. Of the inhibited subjects, six met criteria for at least one anxiety disorder (5 Social Anxiety Disorder, 3 Generalized Anxiety Disorder, 1 Specific Phobia, 1 Anxiety Not Otherwise Specified [NOS]) and two met criteria for Dysthymia (1 comorbid with anxiety). Of the uninhibited subjects, one met criteria for Post Traumatic Stress Disorder and one had comorbid Depression NOS and Anxiety NOS.

\section{Experimental design}

To study temperament differences in expectancy effects, we experimentally manipulated expectation between-subjects within each of the two temperament groups (see Fig. 1). All subjects had previously seen a series of human faces displaying a neutral facial expression during a prior task. For half of the subjects, an expectation was created by stating that they would be seeing "some pictures of people making a scared face" followed by an example picture. The other half of the subjects were told that they would be seeing "some more pictures of faces" and did not see an example, providing a baseline, no expectation, condition. We chose a between-subjects design to maximize the effect of the expectancy manipulation because once the fear faces were shown, future expectations would be changed, especially for the no expectation condition. Assignment to an expectation condition was stratified by temperament and gender. Thus, the design was completely between-groups with Temperament (Inhibited/Uninhibited) and Expectation (Expected/Not Expected) as factors (see Fig. 1).

fMRI task In the fMRI task, subjects passively viewed images of humans displaying a scared facial expression or 
a

\begin{tabular}{lr|c|c|}
\multicolumn{1}{c}{} & \multicolumn{1}{c}{ Expected } & \multicolumn{1}{c}{ Not Expected } \\
\cline { 3 - 4 } Temperament & Inhibited & $\mathrm{N}=9$ & $\mathrm{~N}=9$ \\
\cline { 3 - 4 } Group & Uninhibited & $\mathrm{N}=9$ & $\mathrm{~N}=10$ \\
\cline { 3 - 4 } & &
\end{tabular}

b

Expected Condition

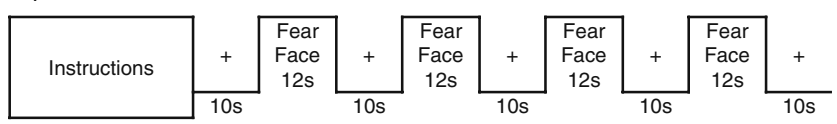

Not Expected Condition

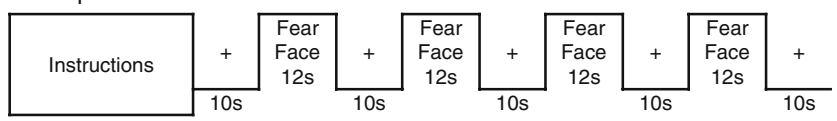

Fig. 1 fMRI task design. We used a block design with four blocks of 12 fear faces per run. a Temperament Group and Expectancy Condition were both between-subjects factors. b Half of each Temperament Group saw the Expected instructions, which read "In this block of trials I am going to show you some pictures of people making a scared face. In a minute, I'll show you an example." and were followed by an example picture. The other half of each Temperament Group saw the Not Expected instructions, which read "In this block of trials I am going to show you some more pictures of faces." and were followed by a blank screen

"fear face". We chose fear faces for our stimuli because facial expressions of fear reliably produce the strongest amygdala activations (Sergerie, Chochol, \& Armony, 2008; Zald, 2003) and are more unfamiliar than other facial expressions (Somerville \& Whalen, 2006). The fear faces were presented in four blocks during a single 98 -s run. We used a block design to increase our power to detect activation differences (Birn, Cox, \& Bandettini, 2002). Similarly, we chose a short session to maximize amygdala activation since the amygdala habituates rapidly with repeated exposure (Breiter et al., 1996; Fischer et al., 2003; Wright et al., 2001). Within the run, there were four 12 -s image blocks, each preceded and followed by a 10-s fixation cross (see Fig. 1). Within each block, 12 faces were presented for $0.5 \mathrm{~s}$ each followed by a $0.5-\mathrm{s}$ interstimulus interval. Faces were randomly arranged within each block for each subject and each face was only presented once.

Stimuli The stimuli were human facial expressions of fear (24 females, 24 males) selected from the Karolinska Directed Emotional Faces database (Goeleven, De Raedt, Leyman, \& Verschuere, 2008; Lundqvist, Flykt, \& Öhman, 1998). Human faces may be especially salient for individuals with inhibited temperament. Previous studies report temperament differences in amygdala response to faces despite intact facial recognition memory (Blackford et al., 2009; Blackford et al., in press). The stimuli were presented in the scanner using Eprime software (Version 1.1, Psychology Software Tools, Pittsburgh, PA). Images were projected onto a screen behind the subject's head and were viewed on a mirror in the scanner.

Data collection

fMRI data acquisition Anatomical and EPI images were collected on 3 Tesla Phillips Achieva magnet (Philips Healthcare, Inc., Best, The Netherlands). High resolution T1-weighted anatomical images were collected $(256 \mathrm{~mm}$ FOV, 170 slices, $1 \mathrm{~mm}, 0 \mathrm{~mm}$ gap). EPI images were acquired using a sequence optimized for the amygdala: $2 \mathrm{~s}$ TR, $22 \mathrm{~ms}$ TE; $90^{\circ}$ flip angle; 1.8 SENSE; $240 \mathrm{~mm}$ FOV; 3 $\times 3 \mathrm{~mm}$ in plane resolution using an $80 \times 80$ matrix (reconstructed to $128 \times 128$ ), and higher-order shimming to limit susceptibility artifacts. Each volume comprised 36 $2.5 \mathrm{~mm}\left(.25\right.$ gap) axial oblique slices (tilted $15^{\circ}$ anterior higher than posterior relative to the intercommisural plane) which provided complete anterior-posterior coverage and inferior-superior coverage from the bottom of the temporal lobe to the top of the most dorsal part of the cingulate gyrus.

fMRI data preprocessing MRI data were pre-processed using SPM5 (http://www.fil.ion.ucl.ac.uk/) and MATLAB (Version 7.1, The MathWorks, Inc, Natick, MA). Data were corrected for motion (aligned to the mean), coregistered to the structural image, normalized into standard stereotactic space (MNI T1 template), resampled to $3 \times 3 \times 3 \mathrm{~mm}$ voxels, and high pass filtered $(128 \mathrm{~s})$. Data were smoothed with a 6-mm FWHM Gaussian kernel to account for individual differences in brain anatomy.

For each subject, motion was assessed and EPI images were visually inspected for artifacts, signal dropout, and coverage of the amygdala. Five subjects had artifacts in the EPI images, caused by scanner issues, and were removed from subsequent analyses (2 Inhibited-Expected, 2 Uninhibited-Expected, 1 Uninhibited-Not Expected).

\section{Data analysis}

fMRI data modeling Using SPM5, a general linear model was estimated for each subject with the fear face blocks as the regressor (Friston et al., 1994). Next, contrast images were created for fear faces minus baseline (fixation cross) for each subject. Contrast images were entered into a full factorial model in SPM5 with two between-subjects factors: Temperament (Inhibited/Uninhibited) and Expectation (Expected/Not Expected). 
Amygdala region of interest The primary analysis focused on the amygdala, based on our a priori hypothesis that the amygdala is a key brain region mediating an inhibited temperament. The bilateral amygdala region of interest was defined using anatomical guidelines specified by the AAL templates (Tzourio-Mazoyer et al., 2002) implemented in WFU Pick Atlas (Version 2.4; Maldjian, Laurienti, Burdette, \& Kraft, 2003). SPM5 was used to test for the interaction of Temperament $\mathrm{X}$ Expectation within the amygdala search region. To control for Type I error due to multiple comparisons, we used a cluster-based threshold. Based on simulations performed with AlphaSim (http://afni. nimh.nih.gov/pub/dist/doc/manual/AlphaSim.pdf), a voxel $\mathrm{p}$-value of .05 and a cluster size of 11 provided a familywise corrected $p<.05$ for each amygdala.

Whole-brain analysis To determine whether there were temperament group differences in expectancy effects on BOLD response in other areas of the brain, we performed an exploratory whole-brain analysis. As with the amygdala analysis, SPM5 was used to test for the interaction of Temperament $\mathrm{X}$ Expectation. For the whole-brain analysis, a voxel $p$ value $<.005$ and a contiguous cluster size of 23 voxels provided a familywise corrected $p<.05$.

Percent signal change For significant clusters in both the amygdala and whole-brain analysis, percent signal change values were extracted for each subject using MarsBar (Brett, Anton, Valabregue, \& Poline, 2002). SPSS (Version 17; SPSS, Inc; Chicago, IL) was used to perform post-hoc analyses (ANOVA and $t$-tests) and to confirm the SPM findings.

\section{Results}

Amygdala results

To determine if the expectancy effects differed between temperament groups, we compared amygdala BOLD signal during viewing of fear faces in persons with an inhibited or an uninhibited temperament who either were or were not expecting to see fear faces. The effect of expectation on amygdala BOLD signal was different for the two temperament groups in both the left and right amygdalae (group $\mathrm{x}$ condition interaction, $p<.05$ corrected; see Fig. 2 and Supplemental Fig. 1). Among subjects with an inhibited temperament who expected to see fear faces, BOLD signal in the left amygdala was significant larger $(p<.05)$ compared to those who were not expecting to see fear faces, but was not significantly
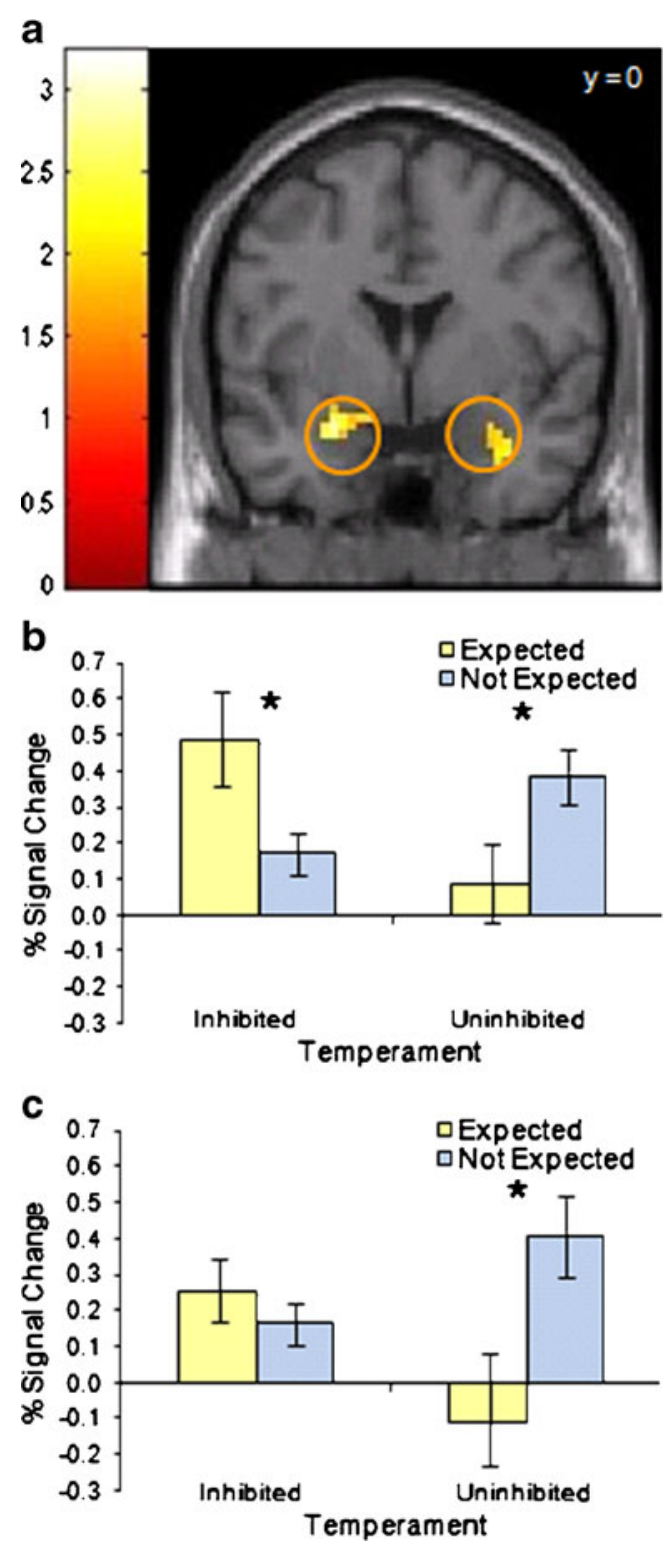

Fig. 2 Amygdala BOLD response is modulated by both temperament and expectation. a For the interaction of Temperament $\mathrm{X}$ Expectation, there were significant clusters ( $p<.05$, corrected) in both the left and right amygdala. Activation maps show $t$-values, threshold at $p<.05$ with cluster size $>11$ voxels and masked to show the amygdala region of interest. The color bar represents $t$ values. b, c Bar graphs show percent signal change values for the left (b) and right (c) amygdala clusters. Error bars represent standard error of the mean. Post-hoc analysis of variance confirmed the interaction for both the left $(p=$ $.003)$ and right $(p=.02)$ amygdala. Asterisks indicate significant differences $(p<.05)$ in post-hoc $t$ tests within each temperament group

different in the right amygdala. By contrast, subjects with an uninhibited temperament who were expecting to see fear faces had smaller amygdalar BOLD signal $(p<.05)$ than uninhibited subjects who were not expecting to see fear faces, in both the left and right amygdalae. There were no main effects of temperament or expectation. 
Whole-brain results

Expectancy effects also differed by temperament group in other brain regions, including dorsal anterior cingulate cortex, dorsomedial prefrontal cortex, inferior frontal gyrus, middle temporal gyrus, and cerebellum (Table 2). For the test of temperament by expectancy, the largest cluster of significant voxels was mainly in an area of the dorsal anterior cingulate cortex (dACC, $p<.05$ corrected, Fig. 3) which included pregenual ACC, supragenual ACC, and also dorsomedial prefrontal cortex. Unlike the pattern in the amygdala, among inhibited subjects BOLD response in the dACC was significantly smaller $(p<.05)$ for those expecting to see fear faces compared to those who were not. Among uninhibited subjects, however, dACC BOLD signal in the Expected group was significantly greater $(p<.05)$ than in the Not Expected group. The interaction of temperament by expectancy in the inferior frontal gyrus and middle temporal gyrus followed the same pattern. In the cerebellum, the findings were similar to those found in the amygdala, with larger BOLD signals among inhibited subjects, in the Expected group, and among uninhibited subjects, in the Not Expected group. There were no main effects of temperament or expectation for any of these brain regions.

\section{Discussion}

The level of expectation for viewing fear faces modulated amygdala and dorsal anterior cingulate cortex (dACC) activation, but the direction of the effects differed in the two temperament groups. In the inhibited temperament group, the amygdalar BOLD response was greater when the subjects expected to see fear faces. This finding provides initial evidence that expectancies critically modulate brain response in people with inhibited temperament by increasing
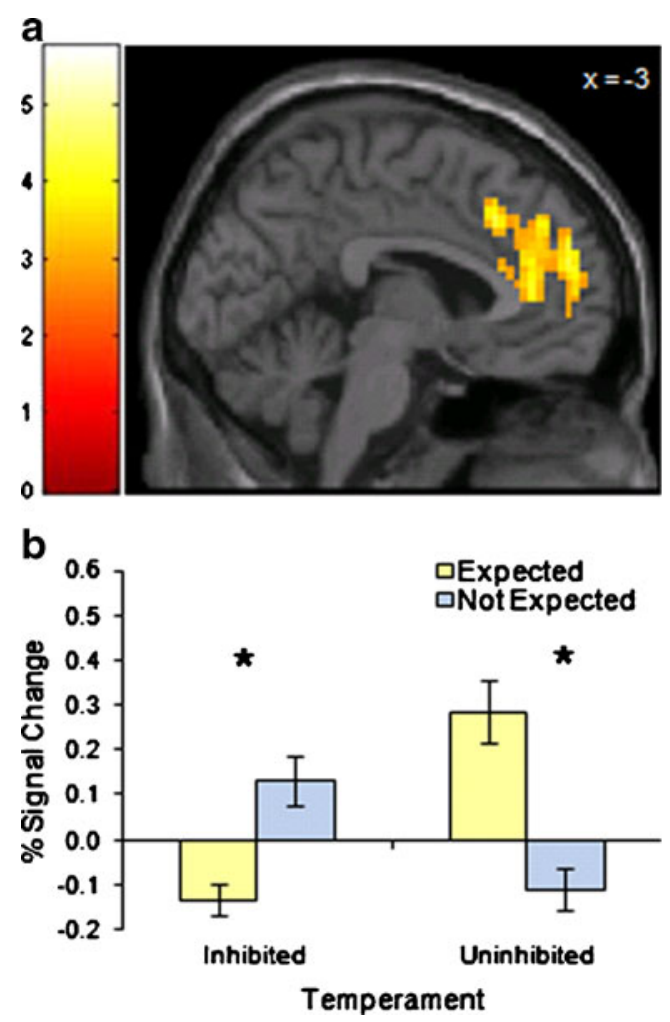

Fig. 3 Dorsal anterior cingulate cortex BOLD response is modulated by temperament group and expectation. For the interaction of Temperament X Expectation, there were significant clusters $(p<.05$, corrected) in the dorsal anterior cingulate cortex. Activation maps show $t$ values, threshold at $p<.005$ with cluster size $>23$ voxels. The color bar represents $t$ values. Bar graphs show percent signal change values and error bars represent standard error of the mean. Post-hoc analysis of variance confirmed the interaction $(p<.001)$. Asterisks indicate significant differences $(p<.05)$ in post-hoc $t$ tests within each temperament group

amygdala response to aversive stimuli. We propose that for persons with inhibited temperament, knowing that an aversive event is imminent may increase vigilance for that event, resulting in an enhanced ability to detect potentially threaten-
Table 2 Brain regions that differed by temperament group and expectation

\begin{tabular}{|c|c|c|c|c|c|}
\hline \multicolumn{2}{|l|}{ Cluster } & \multicolumn{4}{|c|}{ Peak Voxel } \\
\hline \multicolumn{6}{|l|}{ Inhibited $>$ Uninhibited, Expected $>$ Not Expected } \\
\hline Brain Region (Hemisphere) & Cluster Size $\left(\mathrm{mm}^{3}\right)$ & z score & $\mathrm{x}$ & $\mathrm{y}$ & $\mathrm{z}$ \\
\hline Amygdala (L) & 837 & 2.99 & -24 & 0 & -18 \\
\hline Amygdala (R) & 702 & 2.55 & 36 & 3 & -24 \\
\hline Cerebellum declive (L/R) & 1053 & 3.32 & -6 & -78 & -18 \\
\hline \multicolumn{6}{|l|}{ Uninhibited $>$ Inhibited, Expected $>$ Not Expected } \\
\hline Brain Region (Hemisphere) & Cluster Size $\left(\mathrm{mm}^{3}\right)$ & z score & $\mathrm{x}$ & $\mathrm{y}$ & $\mathrm{z}$ \\
\hline $\begin{array}{l}\text { Dorsal anterior cingulate cortex BA } 24,32(\mathrm{~L} / \mathrm{R}) \\
\text { Dorsomedial prefrontal cortex BA 9, } 10\end{array}$ & 14472 & 4.12 & -12 & 45 & 15 \\
\hline Inferior frontal gyrus/Insula BA 44, 45, 47 (L) & 3942 & 4.75 & -42 & 15 & 12 \\
\hline Inferior frontal gyrus/Pars triangularis (L) & 702 & 3.94 & -30 & 33 & 9 \\
\hline Middle temporal gyrus (L) & 702 & 4.10 & -54 & -24 & -9 \\
\hline
\end{tabular}


ing stimuli in the environment. Previous studies support the view that emotional context can modulate physiological response, with startle responses augmented when the emotional valence of the stimulus matches the emotional context, but reduced when they do not match (Lang, Bradley, \& Cuthbert, 1990). In contrast, among people with uninhibited temperament, amygdala activation was stronger when the fear faces were not expected, consistent with prior reports of increased amygdala activation to novel faces (Schwartz et al., 2003b), unexpected images (Bermpohl et al., 2006; Onoda et al., 2008), or unexpected sounds (Koelsch, Fritz, \& Schlaug, 2008).

This study also provides evidence for the dACC as a brain structure that may mediate differences in inhibited temperament. In inhibited subjects, dACC BOLD signal decreased upon exposure to fear faces, whereas for uninhibited subjects, BOLD signal in this region increased when viewing the same stimuli. The $\mathrm{dACC}$ is a brain region implicated in cognitive, inhibitory control over emotional processes (Phillips, Drevets, Rauch, \& Lane, 2003). In typical subjects, dACC is engaged by a variety of tasks including anticipation of viewing aversive images (Herwig, Abler, Walter, \& Erk, 2007; Nitschke, Sarinopoulos, Mackiewicz, Schaefer, \& Davidson, 2006; Onoda et al., 2008), anticipation of pain (Straube, Schmidt, Weiss, Mentzel, \& Miltner, 2009), anticipation of performing a cognitive task (Murtha, Chertkow, Beauregard, Dixon, \& Evans, 1996), aversive conditioning (Buchel, Morris, Dolan, \& Friston, 1998; Buchel, Dolan, Armony, \& Friston, 1999), and viewing expressions of fear (Milad et al., 2007). Hypoactivity in the dACC is associated with fearful temperament in children (Perlman \& Pelphrey, in press), social anxiety disorder (Lorberbaum et al., 2004), and generalized anxiety disorder (Palm, Elliott, McKie, Deakin, \& Anderson, 2010). Increased BOLD signal in the dACC is often inversely correlated with BOLD response in the amygdala (Etkin, Egner, Peraza, Kandel, \& Hirsch, 2006; Hariri, Mattay, Tessitore, Fera, \& Weinberger, 2003; Sarinopoulos et al., 2010). This inverse relationship between the amygdala and dACC is consistent with anatomical projections between the two regions (Bracht, 2009; Carmichael \& Price, 1995; Ghashghaei, Hilgetag, \& Barbas, 2007; Morecraft et al., 2007; Porrino, Crane, \& Goldmanrakic, 1981; Stein et al., 2007), however, see Beckmann, Johansen-Berg, and Rushworth (2009). The strength of the dACC - amygdala relationship is reduced in persons with trait anxiety (Kienast et al., 2008) and high neuroticism (Cremers et al., 2010). We propose that weak inhibitory control by the dACC may contribute to the increased amygdala activation seen in persons with inhibited temperament. Future studies should study the neural substrates of emotion regulation in inhibited temperament.
These data suggest that expectations impact BOLD signal in the amygdala and $\mathrm{dACC}$ when viewing fear faces, and that the effect of expectation is moderated by temperament. However, several critical questions remain. First, we do not know whether the patterns in BOLD response reflect preparatory states or states created by the viewing of the fear faces. Other studies have successfully used a slow event-related design to separately measure BOLD response during anticipation and during viewing (Sarinopoulos et al., 2010). Future studies should measure BOLD response during both a preparatory period and during picture viewing to tease apart the underlying process. Second, while the comparison of two extreme groups provides important information about temperament differences, this approach cannot provide evidence about which of the extreme groups has a dysfunctional response. Findings from other expectancy studies with unselected samples have found a similar pattern to our uninhibited group, suggesting that responses in the inhibited group are dysfunctional, but future studies should include an average temperament group for direct comparison. Third, because neuroimaging analyses are based on contrasts (fear faces minus fixation) it is not possible to determine which part of the contrast is driving the outcome measure. For example, a lower value of percent signal change could represent either small BOLD signal to both fixation and fear face conditions or large BOLD signal to both conditions. Neuroimaging researchers typically assume that response to the fixation cross is similar across groups and conditions, but that is unlikely to be the case. It will be important to develop new experimental designs that test the effects of different baseline conditions in order to better characterize group differences in brain responses.

This study had several limitations. Although the overall study sample size was moderate, sample sizes within each of the four groups were more modest. Also, while we assume that subjects in the baseline condition were expecting to see neutral faces, we did not explicitly set this expectation, leaving open the possibility for other interpretations. However, our findings in the uninhibited group are consistent with expectancy studies directly comparing aversive to neutral images (Bermpohl et al., 2006; Onoda et al., 2008; Sarinopoulos et al., 2010).

A seminal feature of both an inhibited temperament and social anxiety is excessive worry over future social events. Here, the prior expectation of viewing aversive social stimuli increased BOLD responses in the amygdalae among inhibited subjects only, and decreased responses in the dorsal anterior cingulate cortex. Dysfunction in the amygdala-dACC circuit may be one mechanism by which an inhibited temperament confers risk for anxiety disorders. 
Acknowledgements This research was supported in part by funding from the National Institute of Mental Health NIMH (K01-MH083052 to JUB), the National Institute of Drug Abuse (DA015137, DA020149 and DA00366 to RLC), the Vanderbilt Institute for Clinical and Translational Research (1-UL1-RR024975 NCRR/NIH), the Vanderbilt Medical Scholars Program (NIH; 5-TL1-RR024978-04 to JAC), and the Vanderbilt University Institute of Imaging Science. Portions of this work were presented at the Anxiety Disorders Association of America Annual Meeting, Baltimore, MD, 2010. We thank Jerome Kagan for assistance with study design and manuscript preparation; Andy Tomarken and David Zald for helpful comments on the manuscript; and Kristi Simmons and Zak Millman for research assistance.

\section{References}

Bar-Haim, Y., Fox, N. A., Benson, B., Guyer, A. E., Williams, A., Nelson, E. E., et al. (2009). Neural correlates of reward processing in adolescents with a history of inhibited temperament. Psychological Science, 20, 1009-1018.

Beaton, E. A., Schmidt, L. A., Schulkin, J., Antony, M. M., Swinson, R. P., \& Hall, G. B. (2008). Different neural responses to stranger and personally familiar faces in shy and bold adults. Behavioral Neuroscience, 122, 704-709.

Beckmann, M., Johansen-Berg, H., \& Rushworth, M. F. S. (2009). Connectivity-based parcellation of human cingulate cortex and its relation to functional specialization. The Journal of Neuroscience, 29, 1175-1190.

Belova, M. A., Paton, J. J., Morrison, S. E., \& Salzman, C. D. (2007). Expectation modulates neural responses to pleasant and aversive stimuli in primate amygdala. Neuron, 55, 970-984.

Bermpohl, F., Pascual-Leone, A., Amedi, A., Merabet, L. B., Fregni, F., Gaab, N., et al. (2006). Attentional modulation of emotional stimulus processing: an fMRl study using emotional expectancy. Human Brain Mapping, 27, 662-677.

Birn, R. M., Cox, R. W., \& Bandettini, P. A. (2002). Detection versus estimation in event-related fMRI: choosing the optimal stimulus timing. Neuroimage, 15, 252-264.

Blackford, J. U., Avery, S. N., Cowan, R. L., Shelton, R. C., \& Zald, D. H. (in press). Sustained amygdala response to both novel and newly familiar faces characterizes inhibited temperament. Social Cognitive and Affective Neuroscience, doi:10.1093/scan/nsq073.

Blackford, J. U., Avery, S. N., Shelton, R. C., \& Zald, D. H. (2009). Amygdala temporal dynamics: temperamental differences in the timing of amygdala response to familiar and novel faces. $B M C$ Neuroscience, 10, 145.

Blackford, J. U., Buckholtz, J. W., Avery, S. N., \& Zald, D. H. (2010). A unique role for the amygdala in novelty detection. Neuroimage, 50, 1188-1193.

Bracht, T. (2009). Extraction of prefronto-amygdalar pathways by combining probability maps.

Breiter, H. C., Etcoff, N. L., Whalen, P. J., Kennedy, W. A., Rauch, S. L., Buckner, R. L., et al. (1996). Response and habituation of the human amygdala during visual processing of facial expression. Neuron, 17, 875-887.

Brett, M., Anton, J. L., Valabregue, R., \& Poline, J. B. (2002). Region of interest analysis using an SPM toolbox [abstract]. Neuroimage, 16, s497.

Bubic, A., von Cramon, D., \& Schubotz, R. I. (2010). Prediction, cognition and the brain. Frontiers in Human Neuroscience, 4, 25. doi:10.3389/fnhum.2010.00025.

Buchel, C., Dolan, R. J., Armony, J. L., \& Friston, K. J. (1999). Amygdala-hippocampal involvement in human aversive trace conditioning revealed through event-related functional magnetic resonance imaging. The Journal of Neuroscience, 19, 10869 10876.

Buchel, C., Morris, J., Dolan, R. J., \& Friston, K. J. (1998). Brain systems mediating aversive conditioning: an event-related fMRI study. Neuron, 20, 947-957.

Calkins, S. D., Fox, N. A., \& Marshall, T. R. (1996). Behavioral and physiological antecedents of inhibited and uninhibited behavior. Child Development, 67, 523-540.

Carmichael, S. T., \& Price, J. L. (1995). Limbic connections of the orbital and medial prefrontal cortex in macaque monkeys. The Journal of Comparative Neurology, 363, 615-641.

Chronis-Tuscano, A., Degnan, K. A., Pine, D. S., Perez-Edgar, K., Henderson, H. A., Diaz, Y., et al. (2009). Stable early maternal report of behavioral inhibition predicts lifetime social anxiety disorder in adolescence. Journal of the American Academy of Child and Adolescent Psychiatry, 48, 928-935.

Cremers, H. R., Demenescu, L. R., Aleman, A., Renken, R., van Tol, M. J., van der Wee, N. J. A., et al. (2010). Neuroticism modulates amygdala-prefrontal connectivity in response to negative emotional facial expressions. Neuroimage, 49, 963-970.

Essex, M. J., Klein, M. H., Slattery, M. J., Goldsmith, H. H., \& Kalin, N. H. (2010). Early risk factors and developmental pathways to chronic high inhibition and social anxiety disorder in adolescence. The American Journal of Psychiatry, 167, 40-46.

Etkin, A., Egner, T., Peraza, D. M., Kandel, E. R., \& Hirsch, J. (2006). Resolving emotional conflict: a role for the rostral anterior cingulate cortex in modulating activity in the amygdala. Neuron, 51, 871-882.

Fischer, H., Wright, C. I., Whalen, P. J., McInerney, S. C., Shin, L. M., \& Rauch, S. L. (2003). Brain habituation during repeated exposure to fearful and neutral faces: a functional MRI study. Brain Research Bulletin, 59, 387-392.

Foote, S. L., Bloom, F. E., \& Astonjones, G. (1983). Nucleus locus ceruleus - new evidence of anatomical and physiological specificity. Physiological Reviews, 63, 844-914.

Fox, N. A., Henderson, H. A., Rubin, K. H., Calkins, S. D., \& Schmidt, L. A. (2001). Continuity and discontinuity of behavioral inhibition and exuberance: psychophysiological and behavioral influences across the first four years of life. Child Development, 72, 1-21.

Friston, K., Holmes, A., Worsley, K., Poline, J., Frith, C., \& Frackowiak, R. (1994). Statistical parametric maps in functional imaging: a general linear approach. Human Brain Mapping, 2, $189-210$.

Ghashghaei, H., Hilgetag, C. C., \& Barbas, H. (2007). Sequence of information processing for emotions based on the anatomic dialogue between prefrontal cortex and amygdala. Neuroimage, 34, 905-923.

Goeleven, E., De Raedt, R., Leyman, L., \& Verschuere, B. (2008). The Karolinska directed emotional faces: a validation study. Cognition \& Emotion, 22, 1094-1118.

Goodwin, R. D., Fergusson, D. M., \& Horwood, L. J. (2004). Early anxious/withdrawn behaviours predict later internalising disorders. Journal of Child Psychology and Psychiatry, 45, 874-883.

Guyer, A. E., Nelson, E. E., Perez-Edgar, K., Hardin, M. G., RobersonNay, R., Monk, C. S., et al. (2006). Striatal functional alteration in adolescents characterized by early childhood behavioral inhibition. The Journal of Neuroscience, 26, 6399-6405.

Hariri, A. R., Mattay, V. S., Tessitore, A., Fera, F., \& Weinberger, D. R. (2003). Neocortical modulation of the amygdala response to fearful stimuli. Biological Psychiatry, 53, 494-501.

Herwig, U., Abler, B., Walter, H., \& Erk, S. (2007). Expecting unpleasant stimuli - An fMRI study. Psychiatry ResearchNeuroimaging, 154, 1-12.

Hirshfeld, D. R., Rosenbaum, J. F., Biederman, J., Bolduc, E. A., Faraone, S. V., Snidman, N., et al. (1992). Stable behavioral-inhibition and its 
association with anxiety disorder. Journal of the American Academy of Child and Adolescent Psychiatry, 31, 103-111.

Johansen, J. P., Tarpley, J. W., LeDoux, J. E., \& Blair, H. T. (2010). Neural substrates for expectation-modulated fear learning in the amygdala and periaqueductal gray. Nature Neuroscience, 13, 979-U102.

Kagan, J., Reznick, J. S., \& Snidman, N. (1987). The physiology and psychology of behavioral inhibition in children. Child Development, 58, 1459-1473.

Kagan, J., Reznick, J. S., \& Snidman, N. (1988a). Biological bases of childhood shyness. Science, 240, 167-171.

Kagan, J., Reznick, J. S., Snidman, N., Gibbons, J., \& Johnson, M. O. (1988b). Childhood derivatives of inhibition and lack of inhibition to the unfamiliar. Child Development, 59, 1580-1589.

Kagan, J., Snidman, N., Zentner, M., \& Peterson, E. (1999). Infant temperament and anxious symptoms in school age children. Development and Psychopathology, 11, 209-224.

Kienast, T., Hariri, A. R., Schlagenhauf, F., Wrase, J., Sterzer, P., Buchholz, H. G., et al. (2008). Dopamine in amygdala gates limbic processing of aversive stimuli in humans. Nature Neuroscience, 11, 1381-1382.

Koelsch, S., Fritz, T., \& Schlaug, G. (2008). Amygdala activity can be modulated by unexpected chord functions during music listening. NeuroReport, 19, 1815-1819.

Lang, P. J., Bradley, M. M., \& Cuthbert, B. N. (1990). Emotion, attention, and the startle reflex. Psychological Review, 97, 377-395.

Lorberbaum, J. P., Kose, S., Johnson, M. R., Arana, G. W., Sullivan, L. K., Hamner, M. B., et al. (2004). Neural correlates of speech anticipatory anxiety in generalized social phobia. NeuroReport, 15, 2701-2705.

Lundqvist, D., Flykt, A., \& Öhman, A. (1998). The Karolinska Directed Emotional Faces - KDEF. Department of Clinical Neuroscience, Psychology section, Karolinska Institute.

Maldjian, J. A., Laurienti, P. J., Burdette, J. B., \& Kraft, R. A. (2003). An automated method for neuroanatomic and cytoarchitectonic atlasbased interrogation of fMRI data sets. Neuroimage, 19, 1233-1239.

Milad, M. R., Quirk, G. J., Pitman, R. K., Orr, S. P., Fischl, B., \& Rauch, S. L. (2007). A role for the human dorsal anterior cingulate cortex in fear expression. Biological Psychiatry, 62, 446-454.

Morecraft, R. J., Mcneal, D. W., Stilwell-Morecraft, K. S., Gedney, M., Ge, J., Schroeder, C. M., et al. (2007). Amygdala interconnections with the cingulate motor cortex in the rhesus monkey. The Journal of Comparative Neurology, 500, 134-165.

Murtha, S., Chertkow, H., Beauregard, M., Dixon, R., \& Evans, A. (1996). Anticipation causes increased blood flow to the anterior cingulate cortex. Human Brain Mapping, 4, 103-112.

Nebert, D. W. (2000). Extreme discordant phenotype methodology: an intuitive approach to clinical pharmacogenetics. European Journal of Pharmacology, 410, 107-120.

Nitschke, J. B., Sarinopoulos, I., Mackiewicz, K. L., Schaefer, H. S., \& Davidson, R. J. (2006). Functional neuroanatomy of aversion and its anticipation. Neuroimage, 29, 106-116.

Onoda, K., Okamoto, Y., Toki, S., Ueda, K., Shishida, K., Kinoshita, A., et al. (2008). Anterior cingulate cortex modulates preparatory activation during certain anticipation of negative picture. Neuropsychologia, 46, 102-110.

Palm, M. E., Elliott, R., McKie, S., Deakin, J. F. W., \& Anderson, I. M. (2010). Attenuated responses to emotional expressions in women with generalized anxiety disorder. Psychological Medicine. doi:10.1017/S0033291710001455.

Perez-Edgar, K., Roberson-Nay, R., Hardin, M. G., Poeth, K., Guyer, A. E., Nelson, E. E., et al. (2007). Attention alters neural responses to evocative faces in behaviorally inhibited adolescents. Neuroimage, 35, 1538-1546.

Perlman, S. B. \& Pelphrey, K. A. (in press). Regulatory brain development: balancing emotion and cognition. Social Neuroscience.

Phillips, M. L., Drevets, W. C., Rauch, S. L., \& Lane, R. (2003). Neurobiology of emotion perception I: the neural basis of normal emotion perception. Biological Psychiatry, 54, 504-514.
Porrino, L. J., Crane, A. M., \& Goldmanrakic, P. S. (1981). Direct and indirect pathways from the amygdala to the frontal-lobe in rhesusmonkeys. The Journal of Comparative Neurology, 198, 121-136.

Ranganath, C., \& Rainer, G. (2003). Neural mechanisms for detecting and remembering novel events. Nature Reviews. Neuroscience, 4, 193-202.

Reznick, J. S., Hegeman, I. M., Kaufman, E. R., Woods, S. W., \& Jacobs, M. (1992). Retrospective and concurrent self-report of behavioral-inhibition and their relation to adult mental-health. Development and Psychopathology, 4, 301-321.

Sarinopoulos, I., Grupe, D. W., Mackiewicz, K. L., Herrington, J. D., Lor, M., Steege, E. E., et al. (2010). Uncertainty during anticipation modulates neural responses to aversion in human insula and amygdala. Cerebral Cortex, 20, 929-940.

Schwartz, C. E., Snidman, N., \& Kagan, J. (1999). Adolescent social anxiety as an outcome of inhibited temperament in childhood. Journal of the American Academy of Child and Adolescent Psychiatry, 38, 1008-1015.

Schwartz, C. E., Wright, C. I., Shin, L. M., Kagan, J., \& Rauch, S. L. (2003). Inhibited and uninhibited infants "grown up": adult amygdalar response to novelty. Science, 300, 1952-1953.

Schwartz, C. E., Wright, C. I., Shin, L. M., Kagan, J., Whalen, P. J., McMullin, K. G., et al. (2003). Differential amygdalar response to novel versus newly familiar neutral faces: a functional MRI probe developed for studying inhibited temperament. Biological Psychiatry, 53, 854-862.

Sergerie, K., Chochol, C., \& Armony, J. L. (2008). The role of the amygdala in emotional processing: a quantitative meta-analysis of functional neuroimaging studies. Neuroscience and Biobehavioral Reviews, 32, 811-830.

Somerville, L. H., \& Whalen, P. J. (2006). Prior experience as a stimulus category confound: an example using facial expressions of emotion. Social Cognitive and Affective Neuroscience, 1, 271-274.

Spitzer, R. L., Williams, J. B. W., Gibbon, M., \& First, M. B. (1992). The Structured Clinical Interview for DSM-III-R (SCID).1. History, rationale, and description. Archives of General Psychiatry, 49, 624-629.

Stein, J. L., Wiedholz, L. M., Bassett, D. S., Weinberger, D. R., Zink, C. F., Mattay, V. S., et al. (2007). A validated network of effective amygdala connectivity. Neuroimage, 36, 736-745.

Straube, T., Schmidt, S., Weiss, T., Mentzel, H. J., \& Miltner, W. H. R. (2009). Dynamic activation of the anterior cingulate cortex during anticipatory anxiety. Neuroimage, 44, 975-981.

Tzourio-Mazoyer, N., Landeau, B., Papathanassiou, D., Crivello, F., Etard, O., Delcroix, N., et al. (2002). Automated anatomical labeling of activations in SPM using a macroscopic anatomical parcellation of the MNI MRI single-subject brain. Neuroimage, $15,273-289$.

Whalen, P. J. (1998). Fear, vigilance, and ambiguity: Initial neuroimaging studies of the human amygdala. Current Directions in Psychological Science, 7, 177-188.

Windle, M., \& Windle, R. C. (2006). Adolescent temperament and lifetime psychiatric and substance abuse disorders assessed in young adulthood. Personality and Individual Differences, 41, $15-25$.

Wright, C. I., Fischer, H., Whalen, P. J., McInerney, S., Shin, L. M., \& Rauch, S. L. (2001). Differential prefrontal cortex and amygdala habituation to repeatedly presented emotional stimuli. NeuroReport, 12, 379-383.

Wright, C. I., Martis, B., Schwartz, C. E., Shin, L. M., Fischer, H., McMullin, K., et al. (2003). Novelty responses and differential effects of order in the amygdala, substantia innominata, and inferior temporal cortex. Neuroimage, 18, 660-669.

Zald, D. H. (2003). The human amygdala and the emotional evaluation of sensory stimuli. Brain Research Reviews, 41, 88123. 